\title{
Intra-aortic balloon pump as supportive treatment for sepsis-induced cardiomyopathy and cardiogenic shock in a patient with intestinal tuberculosis
}

\author{
E.L. $\underline{\mathrm{Ng}}, \mathrm{MBBS}, \mathrm{MMed}(\text { Anaes)})^{1}$; Lynn $\underline{\text { Yeo }}, \mathrm{MBBS}$, MMed (Anaes), FANZCA 2; Shahla Siddiqui, MBBS, DABA, FCCM ${ }^{3}$ \\ 1 Senior resident, National Health Group, Singapore; ${ }^{2}$ Associate consultant, Khoo Teck Puat Hospital, Singapore \\ 3 Senior consultant, Khoo Teck Puat Hospital, Singapore
}

Background: Sepsis-induced cardiomyopathy $(\mathrm{SIC})^{1}$ is a recognized complication in patients with severe sepsis. It is not known if patients with tuberculosis have a higher risk of SIC and cardiogenic shock ${ }^{2}$. The mainstay of treatment of SIC is treatment of sepsis and inotropic support. The use of intra-aortic balloon pump (IABP) for circulatory support is non-standard and has been rarely reported ${ }^{1}$. We describe a case of successful IABP support of refractory shock in a patient with severe sepsis from perforated intestinal tuberculosis.

Case report: An ASA 138 -year-old female, presented to the emergency department for abdominal pain, diarrhoea, vomiting and fever of one day duration. She was hypotensive and tachycardic on presentation, and was fluid resuscitated in the emergency department.

A computed tomography scan of the abdomen and pelvis showed pneumoperitoneum and ascites with features of peritonitis. There was irregular bowel wall thickening involving the terminal ileum, ileocaecal junction, caecum and ascending colon. This was associated with multiple enlarged necrotic lymph nodes.

The patient underwent emergency laparotomy, right hemicolectomy, small bowel resection and temporary abdominal closure for the perforated caecum. Intraoperatively, she was aggressively fluid resuscitated and required only single vasopressor support with noradrenaline. Noradrenaline requirements escalated in the Intensive Care Unit, and vasopressin and adrenaline infusions had to be added to achieve blood pressure targets. Initial point-of-care transthoracic echocardiography (TTE) in the ICU showed a visual estimated ejection fraction (EF) of 30\%, suggesting septic shock with SIC. A cardiac output monitor (FloTrac) was started in order to guide fluid and vasopressor titration. The patient showed moderate improvement in haemodynamics, and required only noradrenaline by post-op day (POD) 1 . She returned to the operating theatre 24 hours after the first surgery for stoma creation and closure of abdomen.

However, in the following 24 hours, vasopressor and inotrope requirements escalated rapidly again, with refractory hypotension despite high doses of catecholamines.

1. Sato R, Nasu M. A review of sepsis-induced cardiomyopathy. Journal of Intensive Care. 2015;3:48.

2. Sherbini et al Cardiomyopathy Secondary to Tuberculosis: A Case Series And Review Of Literatures Am J Respir Crit Care Med 193;2016:A3777

3. Blanco J, Muriel-Bombin A, Sagredo V, et al. Incidence, organ dysfunction and mortality in severe sepsis: A Spanish multicentre study. Crit Care Med. 2008;12:R158.
This sudden deterioration was attributed to cardiogenic shock secondary to decompensated cardiac failure. Bedside TTE revealed an EF of $10 \%$. An urgent referral to cardiology was made, and the patient was transported to the cardiac catheterisation lab for an insertion of an IABP and a pulmonary artery catheter. A coronary angiogram was done in the same setting, which showed normal coronary arteries, and a pulmonary capillary wedge pressure (PCWP) of $32 \mathrm{mmHg}$. There was drastic haemodynamic improvement with circulatory assistance from the IABP and an improvement of the PCWP to $9 \mathrm{mmHg}$. Catecholamine support was weaned off within 12 hours after insertion of the IABP. The IABP was successfully weaned off and removed 72 hours after insertion. The patient required restarting of noradrenaline and adrenaline infusions post-removal of IABP, as she was still being treated for sepsis and needed a small amount of catecholamine support. TTE on POD 8 showed improvement of EF to $30 \%$, and was extubated on POD 9. She continued to improve and was transferred to the general ward on POD 16.

Discussion: SIC is present in more than $40 \%$ of cases of sepsis. It can increase the mortality rate up to $70 \% 3$. Most patients improve in 7 - 10 days. The IABP has been successfully used for cardiogenic shock due to other causes but rarely in septic patients. By extension, the IABP is suited for this temporary period of support for SIC. Given the drastic improvement after its institution in our patient, it should be considered in the future for selected patients with decompensated SIC.

The need for systemic anticoagulation with an IABP in-situ poses a problem in the post-surgical patient. This problem is compounded when coagulopathy develops as a result of sepsis. The evidence for systemic anticoagulation with IABP has been equivocal, hence it was held off in this patient because of the risk of post-surgical bleeding and coagulopathy from sepsis.

Learning points: Supportive treatment with IABP can be considered for patients with SIC and cardiogenic shock who are insufficiently supported on high dose catecholamines. Point-ofcare TTE can aid in the expeditious diagnosis of SIC and decompensation.

Khoo Teck Puat Hospital

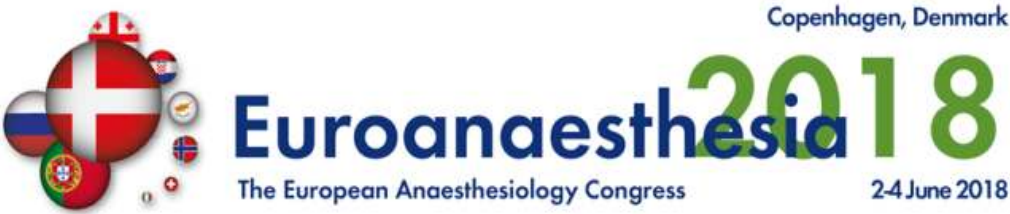

